\title{
PUBLICACIONES CIENTÍFICAS
}

Morales Gil, A. (1969): «Los nuevos regadíos en la Huerta de Murcia». Anales de la Universidad de Murcia, 26, pp. 525-555.

Morales Gil, A. (1969): «El riego con aguas de avenida en las laderas subáridas». Papeles del Departamento de Geografía de la Universidad de Murcia, 1, pp. 137-183.

Calvo García-Tornel, F.; Cano García, G.; López Bermúdez, F.; López Ontiveros, A.; Morales Gil, A.; Moreno Sánchez, J.J.; Ollvares Glaván, C. Roselló I Verger, V.M. (Dir.) (1969): «División comarcal de la provincia de Murcia». Papeles del Departamento de Geografía de la Universidad de Murcia, 2, pp. 9-72.

Morales Gil, A. (1970): «La propiedad rural en el Altiplano de Jumilla-Yecla durante los siglos XVIII y XIX». Papeles del Departamento de Geografía de la Universidad de Murcia, 2, pp. 109-129.

Morales Gil, A. (1971): «Localización industrial en el área urbana de Murcia». Papeles del Departamento de Geografia de la Universidad de Murcia, 3, pp. 159-170.

Morales Gil, A. (1971): «Creación de los Ayuntamientos constitucionales de la Huerta de Murcia en 1820». Murgetana, pp. 29-46.

Morales Gil, A. (1972): El altiplano de Jumilla-Yecla, estudio de geografía comarcal. Murcia, Universidad de Murcia, 469 p.

Morales Gil, A. (1973): «El altiplano de Jumilla-Yecla». En: Conocer España. Salvat, pp. 294-300.

Morales GiL, A. (1974): «Las comunicaciones en Murcia ante el desarrollo económico regional». Papeles del Departamento de Geografía de la Universidad de Murcia, 5, pp. 119-156.

Morales GiL, A. (1974): «Tres ejemplos de centuriato en el Altiplano de JumillaYecla». En Rosello I Verger, V. Ma . (ed.): Estudio de las centuriaciones en España. Madrid, Universidad Autónoma de Madrid, pp. 69-82.

Morales Gil, A. (1975): «La desamortización de bienes de rústica en Hellín». Murgetana, 43, pp. 75-88.

Morales GiL, A. (1976): La vid y el vino en la zona de Jumilla. Murcia, Editorial Academis Alfonso X el Sabio, $150 \mathrm{p}$.

Morales Gil, A. (1976): «Excursión al Altiplano de Jumilla-Yecla». En: Calvo GarcíaTornel, F.; López Bermúdez, F.; Cárdenas Olivares, I.; Morales Gil, A.; Gómez Fayrén, J. F. y Bel Adell, C. Guía de excursiones didácticas de Geografía en el distrito universitario de Murcia. Murcia, Universidad de Murcia, pp. 438-471. 
Morales Gil, A. (1976): «Transformaciones recientes en tres poblaciones del área industrial de Cartagena». En: Actas del IV Coloquio de Geografía. Ciudad e Industria, Asociación de Geógrafos Españoles, pp. 66-83.

Morales Gil, A. (1977): «Consideraciones sobre restos de formas de erosión semiáridas en Lomo de Arico (Tenerife)». En: Morales-Gil, A. (Ed.). Actas V Coloquio de Geografía. Granada, Universidad de Granada, pp. 136-141.

Morales Gil, A. y López Ontiveros, A. (1977): «Los derrubios de ladera en la Sierra del Carche (Murcia)». En: Actas II Reunión Nacional de Geógrafos Españoles. Grupo de Trabajo del Cuaternario. Asociación de Geógrafos Españoles, pp. 137-150.

Morales Gil, A.; Martín Galán, F. y Quirantes González, F. (1977): Formas periglaciares en las cañadas del Teide. Tenerife, Excmo. Cabildo Insular de Tenerife, $110 \mathrm{p}$.

Morales Gil, A. y Martín Galán, F. (1978): «Formas periglaciares en las Cañadas del Teide». En: Actes Colloque Periglaciaire, pp. 79-90.

Morales Gil, A. (1979): «El medio comarcal». En: Roselló Verger, V. (Dir.). Estudio socieconómico de Elche y su comarca. Valencia, Universitat de València, pp. 1-25.

Morales Gil, A. (1979): «Los transportes». En: Roselló Verger, V. (Dir.). Estudio socieconómico de Elche y su comarca. Valencia, Universitat de València, pp. 463-492.

Morales Gil, A. (1980): «Propiedad y tenencia de tierra en el Campo de Cartagena durante la primera mitad del siglo XX». En: Mangas, J.M. (Ed.). La propiedad de la tierra en España. Alicante, Universitat d'Alacant, pp. 103-111.

Morales GIL, A. (1980): «El paisaje rural del campo de Cartagena». En: Los paisajes rurales de España. Fundación Juan March, pp. 287-294.

Morales Gil, A. y JuÁrez Sánchez-Rubio, C. (1982): «Cambios en los usos del agua». Estudios geográficos, 165, pp. 25-40.

Morales Gil, A. (1983): «La red viaria en el área metropolitana de Alicante-Elche». Investigaciones Geográficas, 1, pp. 51-66.

Morales Gil, A.; Bru Ronda, C. y Box Amorós, M. (1983): «Morfología en la umbría de la Sierra de Bernia». Investigaciones Geográficas, 1, pp. 117-145.

Morales Gil, A.; Bru Ronda, C. y Box Amorós, M. (1983): «Las crecidas en los barrancos de las Ovejas y Agua Amarga en octubre de 1982». Estudios geográficos, 170-171, pp. 143-170.

Morales Gil, A.; Bru Ronda, C. y Box Amorós, M. (1983): «Condiciones morfológicas y chubascos de fuerte intensidad horaria en la cuenca vertiente del barranco de las Ovejas (Alicante)». En: Bru Ronda, C.; Gil Olcina, A. y Morales Gil, A. (Eds.). Lluvias torrenciales e inundaciones en Alicante. Alicante, Instituto Universitario de Geografía de la Universidad de Alicante, Servicio de Publicaciones de la Universidad de Alicante, pp. 25-72.

Morales Gil, A. (1984): «La red viaria en el área metropolitana de Murcia». Papeles del Departamento de Geografía de la Universidad de Murcia, 1, 223-230.

Morales Gil, A. y Bru RondA, C. (1984): «Disponibilidades hídricas y modernas técnicas de embalse en el Campo de Alicante». Investigaciones Geográficas, 2, pp. $19-50$. 
Morales Gil, A. (1985): «Accesibilidad por carretera al aeropuerto de Alicante». Canelobre, 5, pp. 73-77.

Morales Gil, A. y ANDrÉs SARASA, J.L. (1985): «Excursión al altiplano Jumilla-Yecla (la transición a la Meseta)». En: Andrés Sarasa, J.L (Dir.). Guía de itinerarios geográficos de la Región de Murcia. Murcia, Universidad de Murcia, pp. 119-135.

Morales Gil, A. (1986): «Problemas referentes al agua en España». En: Actas del IX Congreso de Geógrafos Españoles. Murcia, Universidad de Murcia, pp. 187-196.

Morales Gil, A. (1986): «Los cultivos de secano». En: Uroz Sáez, J. (Ed.). Historia de la provincia de Alicante. Alicante, Mediterráneo Editores, pp. 221-234.

Morales Gil, A. y Box Amorós, M. (1986): «El aprovechamiento del agua y los suelos en un dominio semiárido: la cuenca del Barranco Blanco, Agost (Alicante)». Investigaciones Geográficas, 4, pp. 7-24.

Martín Mateo, R.; Cano, R.; Úbeda, E.; Morales Gil, A. y Vera Rebollo, J.F. (1986): El litoral valenciano y su problemática. Generalitat Valenciana. Valencia, Conselleria d'Obres Públiques, Urbanisme I Transports, 668 p.

Lopéz Bermúdez, F.; Calvo García, F. y Morales Gil, A. (1986): Geografía de la Región de Murcia. Barcelona, Editorial Ketres, 283 p.

Gil Olcina, A.; Morales Gil, A. Bru Ronda, C. y Marco Molina, J.A. (1986): Inundaciones en la ciudad y término de Alicante. Alicante, Universitat d'Alacant, $179 \mathrm{p}$.

Morales Gil, A. (1987): «El agua, un bien escaso». El campo, 103, pp. 31-36.

Morales Gil, A. (1987): «Aprovechamiento conjunto Tajo-Segura y otros trasvases». El campo, 103, pp. 47-50.

Morales Gil, A. (1987): «Lluvias torrenciales e inundaciones del 25 al 26 de julio de 1986 en el término de Jumilla». En: Abellán Pérez, J. (Ed.). Homenaje al Profesor Juan Torres Fontes. Murcia, Servicio de Publicaciones de la Universidad de Murcia, pp. 1.111-1.124.

Morales Gil, A. (1987): «Situación de los transportes de la región murciana en 1986». En: Historia viva de Murcia, 1899-1986. Murcia, Cámara de Comercio, Industria y Navegación de Murcia, pp. 279-292.

García Fernández, J.; Morales Gil, A.; Box Amorós, M. y Marco Molina, J.A. (1987): Contribución al conocimiento geomorfológico del Campo de Alicante. Alicante, Universitat d'Alacant, $101 \mathrm{p}$.

Morales Gil, A. (1988): «Viticultura y vinificación». El campo, 105, pp. 69-73.

Morales Gil, A. (1988): «Usos competitivos del agua en la cuenca del Segura. Investigaciones Geográficas, 6, pp. 10-109.

Morales Gil, A. (1988): «Trasvases de recursos hídricos en España». En: Gil, A. y Morales, A. (Coords.). Demanda y Economía del Agua en España. Alicante, Fundación Cultural de la Caja de Ahorros del Mediterráneo, pp. 239-254.

Gil Olcina, A. y Morales Gil, A. (coords.) (1988): Demanda y Economía del Agua en España. Alicante, Fundación Cultural de la Caja de Ahorros del Mediterráneo, $480 \mathrm{p}$. 
Gil Olcina, A. y Morales Gil, A. (eds) (1988): Prevención de riadas en la provincia de Alicante. Alicante, Instituto Universitario de Geografía de la Universidad de Alicante. Servicio de Publicaciones de la Universidad de Alicante, 800 p.

Morales Gil, A. (1989): «Los cultivos de secano». En: AAVV: Historia de la Provincia de Alicante. Tomo 1-1. Mediterráneo Editores, pp. 221-234.

Morales GiL, A. (1989): «Las condiciones naturales y la circulación en la región de Murcia». En González Blanco, A. (ed.): Los caminos de la región de Murcia: función histórica y rentabilidad socioeconómica. Murcia, Consejería de Pesca Territorial y Obras Públicas, pp. 29-38.

Morales Gil, A. (1989): «Iniciativa popular en la construcción de obras hidráulicas de los siglos XVIII y XIX en Alicante». En: Jean-Louis Miège, J.L.; Perney, M., VillainGandossi, C. (Eds.). L'eau et la culture populaire en la Méditerranée, pp. 31-40.

Morales Gil, A. (1989): «Formas de adaptación al riesgo de avenidas y actuaciones de defensa en la cuenca del río Segura». En: L'uomo e il fiume, pp. 145-152.

Morales GiL, A. (1989): «Abandono y desorganización de los sistemas de riegos de avenida y su incidencia en la escorrentía». En: Los paisajes del agua: Libro jubilar dedicado al profesor Antonio López Gómez. Valencia, Universitat de València, pp. 165-169.

Morales Gil, A. y Vera Rebollo, J.F. (1989): «Ordenación y gestión de recursos hídricos en un ámbito subárido: el abastecimiento de los municipios integrados en la Mancomunidad de los Canales del Taibilla. Investigaciones Geográficas, 7, pp. 51-68.

Morales Gil, A. y Vera Rebollo, J.F. (1989): La Mancomunidad de Canales del Taibilla: Influencia de un gran sistema de abastecimiento público de aguas en el desarrollo económico-territorial. Alicante, Instituto Universitario de Geografía de la Universidad de Alicante. Servicio de Publicaciones de la Universidad de Alicante, $140 \mathrm{p}$.

Morales Gil, A.; Box Amorós, M. y Marco MolinA, J.A. (1989): «El aprovechamiento de las aguas de avenida: Derecho consuetudinario y disposiciones legales». En: Gil Olcina, A. y Morales Gil, A. (Eds.). Avenidas fluviales e Inundaciones en la Cuenca del Mediterráneo. Alicante, Instituto Universitario de Geografía de la Universidad de Alicante. Servicio de Publicaciones de la Universidad de Alicante, pp. 553-564.

Morales Gil, A.; Gil Olcina, A.; Box Amorós, M. y Alberola Romà, A. (1989): Datos para la ordenación del territorio en la cuenca del Montnegre. Alicante, Instituto Universitario de Geografía de la Universidad de Alicante. Servicio de Publicaciones de la Universidad de Alicante, 206 p.

Gil Olcina, A. y Morales GIL. A. (Eds.) (1989): Avenidas fluviales e Inundaciones en la Cuenca del Mediterráneo. Alicante, Instituto Universitario de Geografía de la Universidad de Alicante. Servicio de Publicaciones de la Universidad de Alicante, $586 \mathrm{p}$.

Morales Gil, A. (1990): Alcábala del Viento: Jumilla 1755. Madrid, Tabapress, 158 p.

Morales Gil, A. (1990): "Accesibilidad e infraestructuras de comunicación en el espacio turístico alicantino. Su articulación con el resto del territorio». En: Vera, 
J.F. (Dir.). Libro blanco del turismo en la Costa Blanca II: Estructura territorial y urbanística. Alicante, Cámara oficial de Comercio, Industria y Navegación de Alicante, pp. 100-120.

Morales Gil, A. (1990): «Trasvases en España: distribución y usos de sus caudales». En: Drain, M. (Ed.). Los conflictos territoriales por el agua en los estados nordmediterráneos. Madrid, Universidad Internacional Menéndez Pelayo (UIMP), pp. 84-107.

Morales Gil, A. (Dir.) (1991): Atlas temático de la Comunidad Valenciana. Valencia, Prensa Ibérica, $900 \mathrm{p}$.

Morales Gil, A. (1991): «Alicante, encrucijada de caminos». Investigaciones Geográficas, 9, pp. 55-68.

Morales Gil, A. (1991): «El espacio subregional y ciudades mediterráneas». En: AAVV. Alicante, un proyecto de futuro. Las ciudades mediterráneas en la Europa de los 90. Alicante, Ayuntamiento de Alicante, pp. 196-226.

Morales Gil, A. (1991): «Transporte público y planificación en la escala intermedia». En: Álvarez Vázquez, L. y Navarro Vera, J.R. (Coords.). Tráfico y transporte en la escala intermedia. Alicante, Ayuntamiento de Alicante, pp. 21-37.

Morales Gil, A. (1991): «La inserción territorial de Alicante, una propuesta de nueva centralidad». En: AAVV. Alicante, un proyecto de futuro. Las ciudades mediterráneas en la Europa de los 90. Alicante, Ayuntamiento de Alicante, pp. 27-52.

Morales Gil, A.; Box Amorós, M. y Marco Molina, J.A. (1991): «El medio físico y la presa de Román (Jumilla): un emplazamiento favorable». Investigaciones Geográficas, 9, pp. 69-80.

Morales Gil, A. (1992): «Orígenes de los regadíos españoles: estado de una vieja polémica». En Gil Olcina, A. y Morales Gil, A. (eds): Hitos históricos de los regadíos españoles. Madrid, Ministerio de Agricultura, Pesca y Alimentación, pp. 15-48.

Morales Gil, A. (1992): «Las comarcas y las divisiones comarcales». En: Morales Gil, A. y García Tornel, C. (Eds.). Atlas de la región de Murcia. Murcia, Prensa Ibérica, pp. 373-384.

Morales Gil, A. y Box Amorós, M. (1992): «Los planos de las ciudades». En: Morales Gil, A. y García Tornel, C. (Eds.). Atlas de la región de Murcia. Murcia, Prensa Ibérica, pp. 349-372.

Morales Gil, A. y Calvo García-Tornel, F. (1992): Atlas de la región de Murcia. Murcia, Prensa Ibérica, $420 \mathrm{p}$.

Box Amorós, M. y Morales Gil, A. (1992): «Consecuencias socioeconómicas y medioambientales de los trasvases de agua en España (1978-1992)». Investigaciones Geográficas, 10, pp. 25-36.

Gil Olcina, A. y Morales Gil, A. (eds) (1992): Hitos históricos de los regadios españoles. Madrid, Ministerio de Agricultura, Pesca y Alimentación (MAPA), 415 p.

Morales GIL, A. (1993): Estudio encuesta sobre razones y causas del tráfico de mercancías por el puerto de Alicante y sus conexiones modales con el tráfico de mercancías. Alicante, Cámara oficial de Comercio, Industria y Navegación de Alicante, $220 \mathrm{p}$. 
Morales Gil, A. (1993): «Caracteres geográficos de Murcia». En: Atlas de España. Aguilar Editores, pp. 232-244.

Morales Gil, A. (1993): «Puerto y ordenación del territorio de Alicante». En: Gil Olcina, A.; Campesino, A.; Morales Gil, A.; Vera Rebollo, J.F. y Marchena Gómez, M.J. Algunas cuestiones de ordenación del territorio. Alicante, Instituto Universitario de Geografía de la Universidad de Alicante. Servicio de Publicaciones de la Universidad de Alicante, pp. 41-66.

Morales Gil, A. (1993): «La Comunidad Autónoma de Murcia». En: Crespo Redondo, J. (Ed.). Gran Atlas de España. Aguilar Editores, pp. 446-458.

Morales Gil, A. (1993): «Afinidades agrícolas entre Galicia y el sureste peninsular: intercambios de cultivos». En: Torres Luna, M.P. et al. (Ed.). Los Caminos de Santiago y el Territorio. Xunta de Galicia, pp. 555-566.

Morales Gil, A. (1993): «Las infraestructuras como condicionantes del espacio alicantino». En: Pedreño, A. (Dir.). Estructura económica de la provincia de Alicante. Alicante, Diputación de Alicante, pp. 77-100.

Morales Gil, A. y Box Amorós, M. (1993): "Cambios agrarios en las comarcas de transición del sureste peninsular a La Mancha». En: Gil, A. y Morales, A. (Eds.). Medio siglo de cambios agrarios en España. Alicante, UIMP-Juan Gil Albert, pp. 561-578.

Box Amorós, M. y Morales Gil, A. (1993): «Barrancos y ramblas: su incorporación al entramado urbano en el sureste peninsular». Investigaciones Geográficas, 11, pp. 153-169.

Gil Olcina, A. y Morales Gil, A. (Eds.) (1993): Medio siglo de cambios agrarios en España. Alicante, UimP - Juan Gil Albert, 884 p.

Morales Gil, A. (1994): «Demandas y usos del agua en España». Boletín de la Asociación de Geógrafos Españoles, 18, pp. 3-12.

Morales Gil, A. (1994): «La ordenación del territorio en el sureste peninsular». En: García Fernández, J. (dir). Medio Ambiente y Ordenación del Territorio. Valladolid, Universidad de Valladolid, pp. 125-145.

Morales Gil, A. y Box Amorós, M. (1994): «La laguna del Hondo (Alicante): Humedal y embalse para riego.» En: AAVV. El medio rural español: cultura, paisaje y naturaleza: homenaje a don Ángel Cabo Alonso. Salamanca, Universidad de Salamanca, pp. 181-190.

Morales Gil, A. y Olcina Cantos, J. (1994): «La red ferroviaria en el sureste peninsular: Realidades y propuestas». Papeles del Departamento de Geografía de la Universidad de Murcia, 20, pp. 221-237.

Morales Gil, A. (1995): «Huertas tradicionales, nuevos regadíos y medio ambiente en el valle del Segura». En: García Fernández, J. (dir.). Medio Ambiente y desarrollo rural. Valladolid, Universidad de Valladolid, pp. 131-158.

Morales Gil, A. (1995): «Déficit de agua y demanda de transferencias en la cuenca del Segura». En: Morales Gil, A. y Gil Olcina, A. (Eds.). Planificación hidráulica en España. Alicante, Fundación Cultural de la Caja de Ahorros del Mediterráneo, pp. $378-398$. 
Morales Gil, A. y Gil Olcina, A. (Eds.) (1995): Planificación hidráulica en España. Alicante, Fundación Cultural de la Caja de Ahorros del Mediterráneo, 430 p.

Morales Gil, A. y Marco Molina, J.A. (1995): «Terrazas de cultivo abandonadas en el sureste peninsular: Aspectos evolutivos». Investigaciones Geográficas, 13, pp. 81-92.

Box Amoros, M. y Morales Gil, A. (1995): «Desarrollo urbano y condiciones de escorrentía: adecuación e inadaptación en el sureste peninsular». En: Actas VI Coloquio Ibérico de Geografía. Porto, Universidade do Porto, pp. 1.011-1.015.

Morales Gil, A. y Rico Amorós, A.M. (1996): «Sequías en el sureste de la península ibérica. Cambios en la percepción de un fenómeno natural». Investigaciones Geográficas, 15, pp. 127-143.

Morales Gil, A. (1996): «Escasez y rentabilidad del agua en el Sureste de España». En: García Fernández, J. (Dir.). Medio Ambiente y Crisis Rural. Valladolid, Universidad de Valladolid, pp. 131-158.

Morales Gil, A. (1996): «Planificación peninsular de los usos del agua». En: Fernández, A. y Velasco Bernardo, C. (Eds.). Portugal-España: Ordenación territorial del Suroeste Comunitario. Cáceres, Campesino Universidad de Extremadura, pp. 260-270.

Morales Gil, A. y Box Amorós, M. (1996): «Boqueras y azudes: El aprovechamiento exhaustivo de las aguas esporádicas en el sureste peninsular». En: Alberola, A. (Dir.). Cuatro siglos de técnica hidráulica en tierras alicantinas. Alicante, Instituto de Cultura Juan Gil Albert, Conselleria d'Educació, Cultura I Ciència de la Generalitat Valenciana, pp. 31-42.

Morales Gil, A. (1997): Aspectos geográficos de la horticultura de ciclo manipulado en España. Alicante, Universidad de Alicante, 168 p.

Morales Gil, A.; Rico Amorós, A.M. y Olcina Cantos, J. (1997): «Enseñanzas de la sequía en el sureste ibérico». En: Marzo, M.V.; Dorta, P. y Valladares, P. (Eds.). Clima y agua. La gestión de un recurso climático. Universidad de la Laguna, pp. 211-230.

Morales Gil, A. (1998): «Rareté et rentabilité de l'eau dans le sud-est de l'Espagne: l'agriculture d'avant-garde». Territoires en Mutation, 3, pp. 117-132.

Morales Gil, A. (1998): «Atlas portátil de la región de Murcia». Murgetana, 98, pp. 119-132.

Morales GiL, A. (1998): «El consumo agrícola de agua: sus modalidades y trascendencia socioeconómica actual». En: Gil Olcina, A.; Morales Gil, A. (Eds.). Los usos del agua en España. Alicante, Fundación Cultural de la Caja de Ahorros del Mediterráneo, pp. 49-78.

Gil Olcina, A. y Morales Gil, A. (Eds.) (1998): Los usos del agua en España. Alicante, Fundación Cultural de la Caja de Ahorros del Mediterráneo, 681 p.

Morales Gil, A.; Calvo García-Tornel, F.; Box Amorós, M.; García Escribano, J.J.; Gómez Espín, J.M.; Hernández Hernández, M.; JÍMEnez RodríGuez, A.; López Ruíz, J.M.; Matarredona Coll, E. y Monfort Mir, (Dirs.) (1998): Potencial de captación y generación de tráfico del aeropuerto de Alicante. Madrid, Civitas, 373 p. 
Morales Gil, A. (1999): «José Guardiola Picó, arquitecto higienista». En Estudio preliminar de la obra GuARdiola Picó, J. (1909) Reformas en Alicante para el siglo XX. Tercera parte. Alicante, edición facsímil, COEPA, pp. 59-63.

Morales Gil, A. y Hernández Hernández, M. (2000): «Las infraestructuras viarias en el Valle del Vinalopó». Revista del Vinalopó, 3, pp. 69-85.

Morales GiL, A. (2000): «Articulación Regional: Redes viales». En: Jordá Borrell, R.; Navarro Luna, J.; Miranda Bonilla, J. (Eds.). Hacia un nuevo espacio euromediterráneo. Barcelona, Fundació Catalana per a la Recerca, pp. 171-191.

Morales Gil, A. y Torres Alfosea, F.J. (2000): «Red viaria y organización del territorio». En: Gil, A. (Dir.). Cartografía temática de las tierras alicantinas. Alicante, Instituto Universitario de Geografía de la Universidad de Alicante, pp. 98-105.

Morales Gil, A.; Gil Olcina, A. y Rico Amorós, A.M. (2000): «Diferentes percepciones de la sequía en España: adaptación, catastrofismo e intentos de corrección». Investigaciones Geográficas, 23, pp. 5-46.

Morales Gil, A. (2001): Agua y territorio en la Región de Murcia. Murcia, Fundación Centro de Estudios Históricos e Investigaciones Locales de la Región de Murcia, $270 \mathrm{p}$.

Morales Gil, A.; Olcina Cantos, J y Rico Amorós, A.M. (2001): «Regadíos intensivos». En: Romero, J.; Morales-Gil, A.; Salón, J. y Vera, J.F. (Eds.). La periferia emergente. La Comunidad Valenciana en la Europa de las Regiones. Barcelona, Ariel, pp. 325-342.

Morales Gil, A. (2001): «Secanos y regadíos». En: Gil Olcina, A. y Gómez Mendoza, J. (Eds.). Geografía de España. Barcelona, Ariel, pp. 341-370.

Morales Gil, A. (2001): «Sequías y sobreexplotación de acuíferos en la Submeseta Sur». En: Gil Olcina, A. y Morales Gil, A. (Eds.). Causas y consecuencias de las sequías en España. Alicante, Universidad de Alicante, pp. 389-420.

Morales GiL, A. (2001): «Reestructuración espacial y cualitativa reciente del viñedo de la región de Murcia». En: Manero, F. (ed.). Espacio natural y dinámicas territoriales. Homenaje al Dr. D. Jesús García Fernández. Valladolid, Universidad de Valladolid, pp. 503-514.

Morales Gil, A. (2001): «Recursos naturales y fuentes de energía». En: Romero, J.; Morales Gil, A.; Salón, J.; Vera, J.F. (Eds.). La periferia emergente. La Comunidad Valenciana en la Europa de las Regiones. Barcelona, Ariel, pp. 83-93.

Morales GiL, A. (2001): «Sistema de comunicaciones y accesibilidades». En: Romero, J.; Morales Gil, A.; Salón, J.; Vera, J.F. (Eds.). La periferia emergente. La Comunidad Valenciana en la Europa de las Regiones. Barcelona, Ariel, pp. 94-114.

Gil Olcina, A. y Morales, A. (Eds.) (2001): Causas y consecuencias de las sequías en España. Alicante, Universitat d'Alacant, 574 p.

Romero, J.; Morales, A.; Salón, J. y Vera, J.F. (2001): La periferia emergente. La Comunidad Valenciana en la Europa de las Regiones. Barcelona, Ariel, 587 p.

Morales GiL, A. (2002): «El riego con aguas de avenida en las laderas subáridas». En: Palerm Viquiera, J. y Martínez Saldaña, J. (Eds.). Antología sobre pequeño riego. Volumen II: sistema de riego no convencionales. Editorial Plaza y Valdés (México), pp. 117-136. 
Morales Gil, A. (2002): «Rentabilidad del agua para usos agrarios». En: Gil Olcina, A. y Morales Gil, A. Insuficiencias hídricas y Plan Hidrológico Nacional. (Eds.). Alicante, Universitat d'Alacant, pp. 149-178.

Morales Gil, A. (2002): «Un modelo de eficiencia en el abastecimiento urbano de agua: La Mancomunidad de Canales del Taibilla». En: Cánovas, J. y Melgarejo, J. (Eds.). La Confederación Hidrográfica del Segura 1926-2001. 75 Aniversario. Madrid, Ministerio de Medio Ambiente (MIMAM), pp. 289-310.

Morales Gil, A. (2002): «Recursos naturales y fuentes de energía». En: Morales-Gil, A.; Romero, J.; Salom, J. y Vera, J.F. (Eds.). Comunidad Valenciana. Barcelona, Ariel, pp. 45-50

Morales GIL, A. (2002): «El sistema de comunicaciones y accesibilidad». En: MoralesGil, A.; Romero, J.; Salom, J. y Vera, J.F. Comunidad Valenciana. Barcelona, Ariel, pp. 51-58.

Morales Gil, A.; Olcina Cantos, J. y Rico Amorós, A.M. (2002): «Regadíos intensivos». En: Morales-Gil, A.; Romero, J.; Salom, J. y Vera, J.F. Comunidad Valenciana. Barcelona, Ariel, pp. 133-140.

Morales Gil, A.; Olcina Cantos, J y Rico Amorós, A.M. (2002): «Aspectos cualitativos de las sequías». En: Ayala-Carcedo, F.J. y Olcina Cantos, J. (coord.). Riesgos Naturales. Barcelona, Ariel, pp. 575-604.

Morales Gil, A.; Romero, J.; Salom, J. y Vera, J.F. (2002): Comunidad Valenciana. Barcelona, Ariel, $291 \mathrm{p}$.

Gil Olcina, A. y Morales Gil, A. (Eds.) (2002): Insuficiencias hídricas y Plan Hidrológico Nacional. Alicante, Universitat d'Alacant, 512 p.

Morales Gil, A. (Ed.) (2003): Cultura, paisajes y sociedades en el eje de desarrollo territorial del Bajo Segura y Campo de Cartagena. Murcia, AUSUR, 390 p.

Morales Gil, A. (2003): «Eficiencia de los regadíos españoles». Cuadernos de Geografía, 73/74, pp. 323-342.

Morales Gil, A. (2003): «Articulación funcional: accesibilidad y redes viales de comunicación». En: Morales Gil, A. (dir). Cultura, paisajes y sociedades en el eje de desarrollo territorial del Bajo Segura y Campo de Cartagena. Murcia, AUSUR, pp. 195-220.

Rico Amorós, A.M. y Morales Gil, A. (2003): «Regadíos hortícolas y frutícolas». En: Morales Gil, A. (dir.). Cultura, paisajes y sociedades en el eje de desarrollo territorial del Bajo Segura y Campo de Cartagena. Murcia, AUSUR, pp. 221-246.

Morales GIL, A. (2004): «Significado y valor de las actividades extractivas en la región de Murcia». Boletín de la Asociación de Geógrafos Españoles, 38, pp. 74-84.

Morales Gil, A. (2004): «Trascendencia territorial del puerto de Cartagena». Investigaciones Geográficas, 33, pp. 107-118.

Morales GIL, A. (2004): «La incorporación de ramblas y barrancos a las tramas urbanas en tierras alicantinas». En: Gil Olcina, A.; Olcina Cantos, J. y Rico Amorós, A.M. (Eds.). Aguaceros, aguaduchos e inundaciones en áreas urbanas alicantinas. Alicante, Universidad de Alicante, pp. 119-124. 
Morales GiL, A. (2004): «Las hortalizas». En: Molinero Hernándo, F.; Majoral Moline, R. el al. (Dirs.). Atlas de la España Rural. Madrid, Ministerio de Agricultura, Pesca y Alimentación (MAPA), pp. 224-227.

Morales Gil, A. (2004): «Los frutales». En: Molinero Hernándo, F.; Majoral Moline, R.; García Bartolomé, J.M. y García Fernández, G. (Dirs.). Atlas de la España Rural. Madrid, Ministerio de Agricultura, Pesca y Alimentación (MAPA), pp. 228-232.

Morales GiL, A. (2004): «Horticultura de ciclo manipulado y citricultura selecta». En: Gil, A.; Morales, A.; Calvo, F., Amor, F.; Courtot, R.; Herin, R.; Rico, A.M. y Olcina, J. (Dirs.). Aridez, salinización y agricultura en el sureste ibérico. Madrid, Centro de Estudios Ramón Areces, S.A., pp. 36-68.

Morales Gil, A. (2004): «Aprovechamiento de aguas turbias». En: Gil Olcina, A. (dir.). La Cultura del Agua en la cuenca del Segura. Murcia, Fundación CajaMurcia, pp. 403-438.

Morales Gil, A. (2004): «Luz, temperatura, viento y humedad, factores básicos para la localización de la horticultura de ciclo manipulado en España». En: Historia, clima y paisaje. Estudios Geográficos en memoria del profesor Antonio López Gómez. Valencia, Departamento de Geografía de la Universidad de Valencia, pp. 529-538.

Morales Gil, A. (2004): «Evolución y distribución territorial de las demandas de agua para usos agrícola y su incidencia en el régimen de los ríos». En: Gil Olcina, A. (Ed.). Alteración de los regímenes fluviales peninsulares. Murcia, Fundación CajaMurcia, pp. 99-122.

Gil Olcina, A.; Morales Gil, A. y Torres Alfosea, F. J (coord.) (2004): Aridez, salinización y agricultura en el sureste ibérico. Madrid, Centro de Estudios Ramón Areces, S.A., 255 p.

Morales Gil, A. (2005): «Formas productivas y agua en Murcia». En: Romero, J. y Alberola, M. (coords.). Los límites del territorio. El País Valenciano en la encrucijada. Valencia, Universitat de València, pp. 227-230.

Morales Gil, A.; Rico Amorós, A.M.; Hernández Hernández, M. (2005): «El trasvase Tajo-Segura». Observatorio Medioambiental, 8, pp. 73-110.

Morales Gil, A. (2006): «Usos tradicionales del suelo y riesgo de avenidas en las tierras del litoral mediterráneo español». En: Alberola, A.; Drain, M. et al. (Dirs.). Riesgo de inundaciones en el Mediterráneo occidental, pp. 131-155.

Morales Gil, A. (2007): «Espacios de relación». En: Delgado Viñas, C.; Frochoso Sánchez, M.; González Pellejero, R.; González Urruela, E.; De Meer Lecha-Marzo, A.; De la Puente Fernández, L. y Reques Velasco, P. (coords.). Espacios públicos, espacios privados. Un debate sobre el Territorio. Ponencias XIX Congreso de Geógrafos Españoles. Asociación de Geógrafos Españoles, pp. 131-185.

Hernández Hernández, M. y Morales Gil, A. (2007): «La evolución de la agricultura de regadío en el Alto y Medio Vinalopó»». En: Hermosilla Pla, J. (Dir.). Los regadios tradicionales del Vinalopó (Allto y Medio). Valencia, Dirección General de Patrimonio Cultural Valenciano y Museos, pp. 37-54.

Morales GiL, A. (2008): «Trade flows in Spanish Mediterranean port areas». En: Alario Trigueros, M. (coord.). España y el Mediterráneo. Una reflexión desde la Geografía 
Española. Comité español de la Unión Geográfica Internacional. Asociación de Geógrafos Españoles, pp. 98-104.

Hernández Hernández, M. y Morales Gil, A. (2008): «Trascendencia socio-económica del trasvase Tajo-Segura tras 30 años de funcionamiento en la provincia de Alicante». Investigaciones Geográficas, 46, pp. 31-48.

Morales GIL, A. (2009): «Los grandes complejos portuarios españoles mediterráneos y su integración en el transporte marítimo global». En: Calvo García Tornel, F. (coord.). Homenaje al Académico Miguel Ortuño Palao. España, Academia Alfonso X el Sabio, pp. 203-228.

Hernández Hernández, M. y Morales Gil, A. (2009): «La hortofruticultura y las aguas del trasvase Tajo-Segura: repercusiones socioeconómicas». En: Melgarejo Moreno, J. (Dir.). El Trasvase Tajo-Segura: Repercusiones económicas, sociales y ambientales en la cuenca del Segura. CAM Cultural, pp. 413-464.

Morales GiL, A. (2010): «Las superficies y la organización espacial de redes de transporte de mercancías en España». Papeles de Geografía, 51-52, pp. 211-222.

Morales Gil, A. y Hernández Hernández, M. (2010): «Mutaciones de los usos del agua en la agricultura española durante la primera mitad del siglo XXI». Investigaciones Geográficas, 51, pp. 27-52.

Hernández Hernández, M. y Morales Gil, A. (2012): «Patrimonio agrario: paisaje y cultura en las riberas del Mediterráneo». En: Barciela, C.; López, M., Inmaculada; Melgarejo, J. (Dirs.). Los Bienes Culturales y su Aportación al Desarrollo Sostenible. Alicante, Secretariado de Publicaciones de la Universidad de Alicante, pp. 263-290.

Hernández Hernández, M. y Morales GiL, A. (2013): «Los aprovechamientos tradicionales de las aguas de turbias en los piedemontes del sureste de la península Ibérica: estado actual en tierras alicantinas». Boletín de la Asociación de Geógrafos Españoles, 63, pp. 105-123.

Morales Gil, A. (2014): «Reflexiones sobre estímulos y carencias actuales de la horticultura española». En: Olcina Cantos, J. y Rico Amorós, A.M. (coords.). Libro jubilar en homenaje al profesor Antonio Gil Olcina. Alicante, Servicio de Publicaciones de la Universidad de Alicante, pp. 631-651.

Hernández Hernández, M.; Morales Gil, A. y Saurí Pujol, D. (2014): «Ornamentals Plants and the production of nature(s) in the Spanish real boom and burst: the case of Alicante». Urban Geograhpy, 35, pp. 71-85.

Hernández Hernández, M.; Saurí Pujol, D. y Morales Gil, A. (2015): «Auge y caída de nuevas naturalezas urbanas: plantas ornamentales y expansión turísticoresidencial en Alicante». Boletín de la Asociación de Geógrafos Españoles, 68, pp. 129-157.

Márquez, J.A.; Gordo, M. y Morales, A. (2015): «El laberinto jornalero. Evolución reciente de extranjeros y españoles en la agricultura». En: Márquez Domínguez, J.A. (Dir.). MHs all de la competencia agrícola hispano-marroquí. La Gestión colectiva en contratos en origen. Huelva, Servicio de Publicaciones de la Universidad de Huelva, pp. 129-182. 